\title{
Calculation Of Blade Track Height Using An Optical Tracker
}

\author{
Eric Bechhoefer ${ }^{1}$, and Brian Tucker ${ }^{2}$ \\ ${ }^{1}$ CEO/Chief Engineer GPMS Inc., Cornwall, VT, 05753, USA \\ eric@gpms-vt.com \\ ${ }^{2}$ Associate Technical Fellow, Bell Flight, Ft Worth, TX, 76118, USA \\ btucker4@bellflight.com
}

\begin{abstract}
Rotor Track and Balance (RTB) is a necessary maintenance action to improve aircraft ride quality and decrease fatigue for both the aircrew and helicopter equipment. It is required maintenance when blades are changed. While there have been a number of papers discussing the solution strategy for optimizing both blade track split and vibration, little work has been reported on how to actually calculate blade track with using an optical sensor. This paper will discuss the formulation of the blade track height estimate based on optical tracker measurements from flight-testing. An optical track typically uses the difference in time between the time of a blade's leading between the field of view of two light sensitive diodes. An enhanced optical tracker also measures the time between the leading and trailing edge of a blade. This paper will include a sensitivity analysis to define the blade track height error between the two-pulse and threepulse optical trackers.
\end{abstract}

\section{INTRODUCTION TO RTB}

In general, the RTB maintenance procedure is to track and balance the main rotor. This is required after: any component changes to the main rotor hub or blade assembly, after any pitch control link (PCL) replacement, after any major overhaul of the helicopter, or if the flight crew reports a rough ride.

Beside reducing crew fatigue, vibration is damaging to the airframe and avionic equipment. Veca's (1973) seminal study of the effect of vibration on helicopter reliability showed that reducing vibration significantly improves reliability and reduce maintenance and life-cycle costs. This in turn resulted in the development of a number of RTB system to assist in the maintenance of improving track and

Eric Bechhoefer et al. This is an open-access article distributed under the terms of the Creative Commons Attribution 3.0 United States License, which permits unrestricted use, distribution, and reproduction in any medium, provided the original author and source are credited. reducing vibration in the aircraft.

The RTB phenomenology is complex. There is not direct relationship between track and vibration: it is widely acknowledged that having a "flat track" (e.g. distance between the maximum and minimum blade track) does not necessarily ensure low vibration. Typically, the aircraft is subject to lateral and vertical vibration. Lateral vibration is due to the unequal distribution of mass in the rotor disk. This can be correct through adding "weights" to the main rotor hub or through "sweeping" the blade. Sweeping (e.g. moving the blade fore or aft of its angular position), in turn affects center of mass.

Vertical vibration is caused by unequal lift produced by the rotor blades. This vibration can be controlled by changing the length of the PCL (which changes the blade angle of attack), or by moving a trim tab (TAB) on the blade. PCLs are used to reduce track split in ground and hover, while TABs (whose effectiveness increases with forward flight speed), are used to control track split in forward flight. Note that by changing the blade track (with either PCL or TAB adjustment), the blades drag will change.

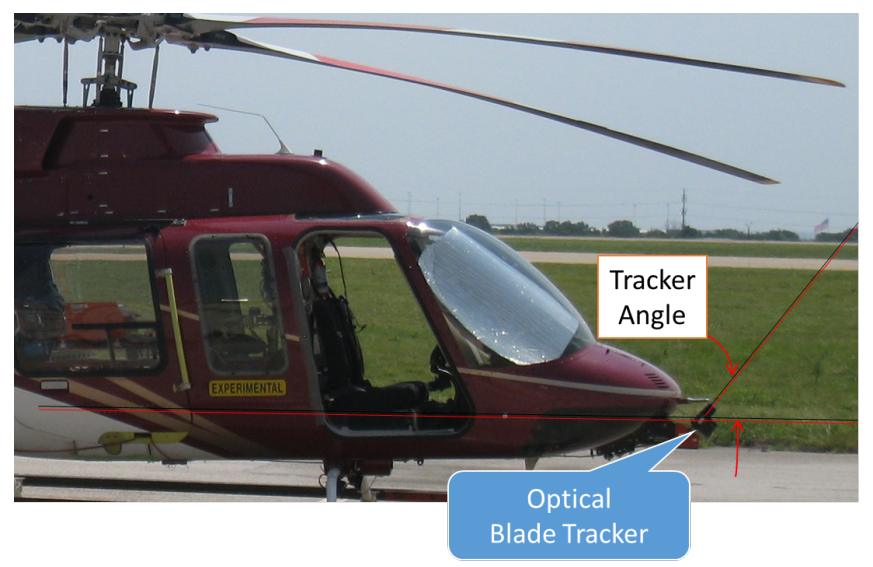

Figure 1 Optical Tracker Installed on an Bell 407GX

The effect of reduced drag causes the blade to "lead", while increasing drag, "lags" the blade. Hence PCR and TAB 
adjustments are coupled to center of mass/lateral changes in vibration. RTB is typically a two-stage process of first flattening track, then reducing vibration. This process is done for various regimes which are set by the manufactures, such as: Ground, Hover, 60 Knots, 100 Knots, 120 Knots, 130 Knots and Vne. The while track height is typically measured at the waterline of the aircraft, vibration is measured at a number of sensors, such a cabin vertical, cockpit vertical and cockpit horizontal locations (Aviation Pros, 1999).

\section{Track Measurement}

The track measurements were taken using an Enhanced Universal Tracking Device (EUTD) manufactured by GE Aviation. This precision instrument uses two diodes to trigger an open collector (NPN) interface. The EUTD diodes have a field of view of 11.43 degrees. As the leading edge of the rotor blade passes the field of view of diode 1 (d1), the EUTD goes from low to high, with a pulse width of 50 $\mu \mathrm{s}$. As the leading edge of the rotor blade passes the field of view of diode 2, (d2), the EUTD generates a second pulse. Finally, when the trailing edge of the rotor blade passes $\mathrm{d} 2$, a third pulse is generated (Figure 2).
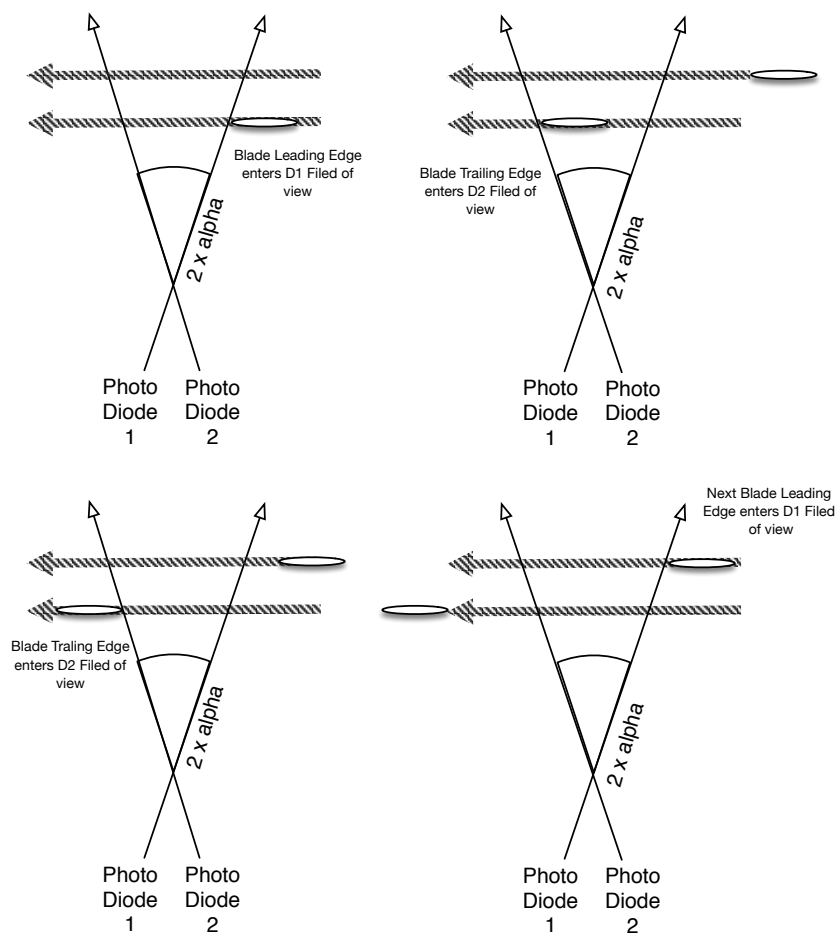

Figure 2 Blade Tracker Timing Example

The time between trigger 1 and trigger 2 is proportional to the blade track height. In this example, the blade one delay between the leading and trailing edge is: $17.867067 \mathrm{msec}$. The time between trigger 2 and trigger 3 is proportional to blade cord: $1.04759 \mathrm{msec}$ (Figure 3). Essentially, the EUTD is a tachometer, whose trigger is based on the change in contrast of the blade as it passes the sensor. The EUTD interfaces into a clock, which measures the time between pulses. In order to calculate phase (e.g. which blade has passed the sensor), a key phase time is needed for a $1 / \operatorname{Rev}$ indicator.

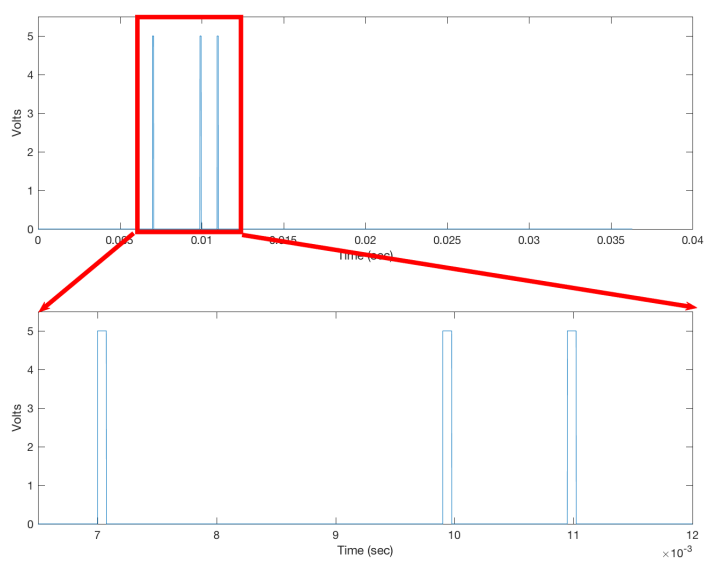

Figure 3 EUTD Pulse Example

The installed system for gathering both vibration and track data was the Foresight MX: a bused, condition monitoring system. Figure 3 shows the system used to gather RTB data in this study. This bused system architecture performs data acquisition in the smart sensors and uses a digital data bus to transfer the data within the system. This approach offers a lower cost and weight solution when compared to star network system architectures. The system consisted of an on board control unit (OBCU) which is powered by aircraft utility power (upper left), bus interface units (" $1 /$ Rev Tach, unit and "EUTD Interface" unit), smart sensors to collect vibration (Vert Cabin, Vert Cockpit, and Horizontal Cockpit), an optical tracker (EUTD), and azimuth sensor ("1/Rev Key Phase"). Both the $1 /$ Rev Tach and EUTD Interface use a $48 \mathrm{MHz}$ clock to accurately measure the transition from low to high (EUTD) or zero cross $(1 / \mathrm{Rev}$ Key Phase) times. Note that each sensor runs it own clock.

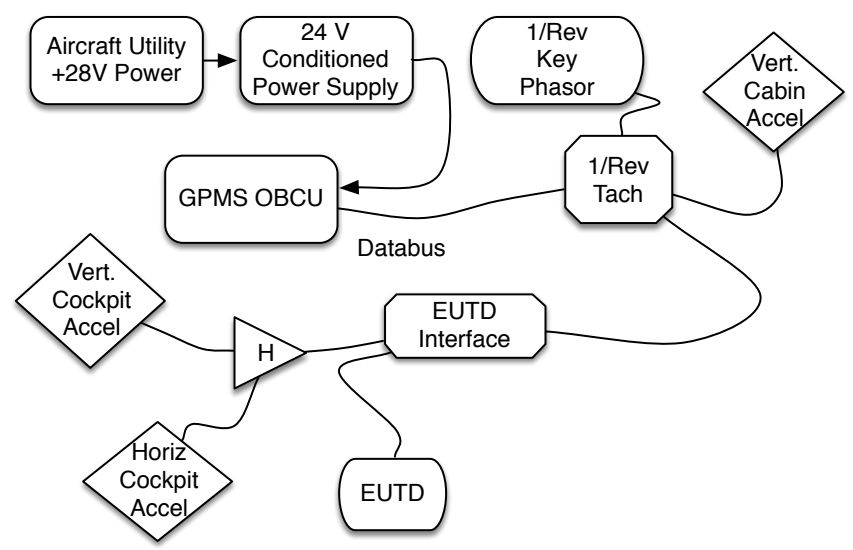

Figure 4 Data Acquisition System 
Because there are two distinct clocks, which are triggered by the OBCU, there is a need to quantify the phase error between each bused interface. Possible sources of errors between the clocks are:

- At the start of an acquisition, there could be random error due to each interface RTOS (real time operating system) being in different states as it waits for the acquisition command.

- After the start acquisition command, there is some small amount of time needed for the hardware to respond and start the interrupt timer, which measures the rising edge of the sensors.

A test was run where the EUTD interface and Tachometer $(1 / \mathrm{Rev})$ interface were triggered off of a common source. This allowed a measure of the between system and within system timing delays. The distribution of the two interfaces, based on a sample size of 37 trials, was a bias of $8 \mathrm{e}-5$ seconds, and RMS of 7e-4 seconds. It is likely that the bias, being 0.1 of the RMS, is effectively zero, e.g. there is not enough evidence to support the alternative hypothesis that the bias is not zero.

As the EUTD is, essentially, a tachometer that measures multiple targets (e.g. the leading edge of the blade passing $\mathrm{d} 1$, the leading edge of the blade passing $\mathrm{d} 2$, and the trailing edge of the blade passing $\mathrm{d} 2$ ), one can directly compare the calculated main rotor shaft rate to the $1 / \mathrm{Rev}$ striker by decimating the data (taking every Number of blades $\mathrm{x} 3$ data points). The shaft rate is simply:

$$
\text { shaft rate }=1 / \operatorname{diff}(\text { zero cross times) }
$$

Figure 4 shows a comparison of evolution of main rotor shaft rate over time for the $1 / \mathrm{Rev}$ tachometer and the EUTD, using data from the Blue blade. The mean absolute error between the two signals is 0.0012 percent, or $1.19 \mathrm{e}-5 \mathrm{~Hz}$.

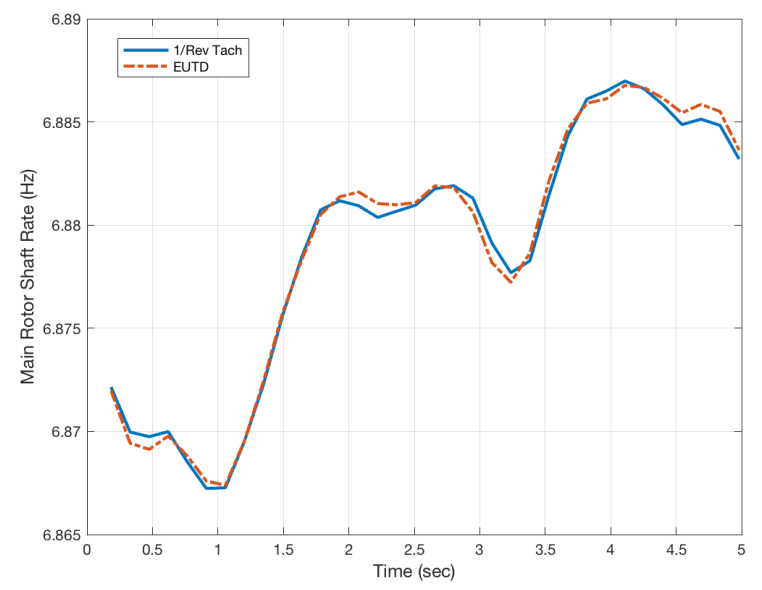

Figure 5 Comparison of Shaft Rates calculated from the EUTD and the 1/Rev tachometer
For a main rotor shaft of $6.88 \mathrm{~Hz}$ (413 RPM), this suggest that $68 \%$ of the time, the phase error between the $1 / \mathrm{Rev}$ tachometer and the EUTD phase is less than 1.7 degrees.

\section{Calculation of Track}

Consider the cartoon in Figure 5. The left hand side of the figure is a side view of the aircraft and shows the blade at the top turning around the axis of rotation. The tracker is displaced horizontally and vertically from the rotor hub by distances of $\mathrm{X}$ and $\mathrm{Y}$ respectively.

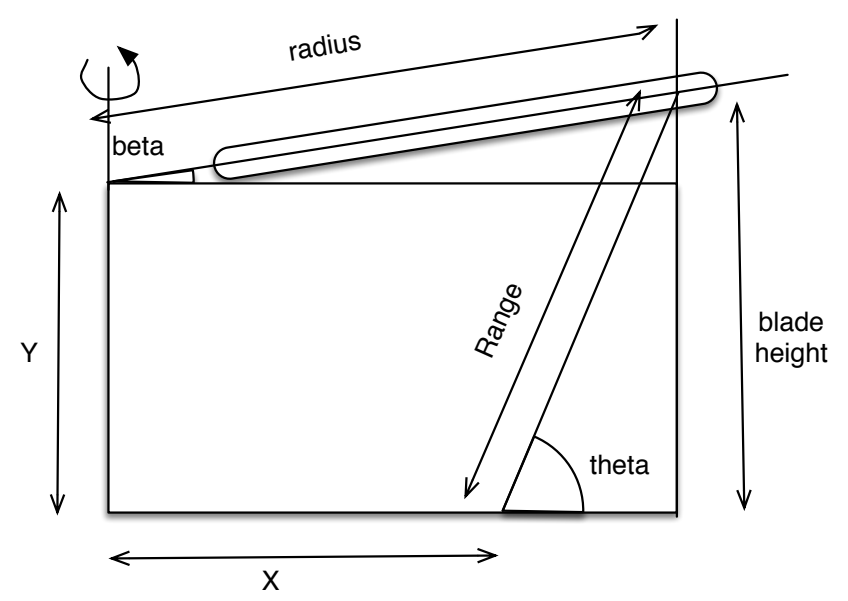

Figure 6 Cartoon of Aircraft Tracker Layout

The tracker is mounted at an angle $\theta$ (in this case between 50 and 60 degrees as per the mainteance manual (2014)), which determines the point where the field of view of the tracker will intersect the blade. To the right of the figure and representing a view of the front of the aircraft, the optical tracker photodiodes have a narrow field of view along lines $\mathrm{d} 1$ and $\mathrm{d} 2$ (which are displaced by angle $\alpha$, 11.43 degrees, from the aircraft vertical axis). When a blade initially transects the field of view of a photodiode, a pulse is generated. As noted previosuly, the time between the pulse $\mathrm{d} 1$ and $\mathrm{d} 2$ are proportional to blade track height.

From Figure 5, it can be seen that the range from the tracker to the blade tip is:

and

$$
\text { radius } * \sin (\beta)+Y=\text { Range } * \sin (\theta)
$$

$$
\text { radius } * \cos (\beta)=\text { Range } * \cos (\theta)+X \text { (eq } 3)
$$

Given a blade angular velocity of $\Omega$, and a time interval, $T_{12}$, between diode $1(\mathrm{~d} 1)$ and diode $2(\mathrm{~d} 2)$, then the relationship is:

$$
\Omega * T_{12} * \text { radius } * \cos (\beta)=2 * \text { Range } * \tan (\alpha)(\text { eq } 4)
$$

The blade height, $H$, is then:

$$
H=\text { Range } * \sin (\theta)
$$

From the trigonometric identity, 


$$
\sin (\theta)=\sqrt{1-\cos ^{2}(\theta)}
$$

assuming $\cos (\beta)$ near 1 , it follows that,

$$
H=\frac{\Omega * T_{12} * \text { radius }}{2 \tan (\alpha)} \sqrt{1-\left[\frac{(\text { radius }-X) 2 \tan (\alpha)}{\Omega * T_{12} * \text { radius }}\right]^{2}}
$$

Using measurements from the tachometer $1 /$ Rev striker, the angular velocity can be calculated, while the time $T_{12}$ is calculated from the EUTD difference in time from $\mathrm{d} 2$ to $\mathrm{d} 1$. The value, $\mathrm{X}$ (horizontal displacement of the optical track to the rotor mast) was calculated from the aircraft drawing, as $3.27 \mathrm{~m}$. The tracker view angle, $\alpha$ : the tracker view angle / 2 , was given at 11.43. Finally, what remains to solve for $\mathrm{H}$ is the radius of the blade where the view of the tracker intersects the blade.

\subsection{The Issue of Radius Error}

The radius of the Bell 407 blades is $5.33 \mathrm{~m}$. The optical tracker does not view the blade tip, but at some intermediate range which is a function of the mounted angle: between 50 and 60 degrees. This means that the measured radius will change depending on what angle the maintainer the optical tracker attached. Further, the tools for measuring track blade height are generalized. That is, there is a software configuration using a nominal radius for a give platform. Hence, this lack of radius information is a source of error in the calculation of blade tip height, as radius is dependent on how the tracker is mounted.

Consider that the actual radius of the blades where viewed by the tracker is $4.7 \mathrm{~m}$. Given $\mathrm{X}=3.27 \mathrm{~m}$, then the blade height is:

$$
H=(4.7-3.27) \times \tan (\theta)
$$

The blade height $\mathrm{H}$ for $\theta=50$ degrees is $1.65 \mathrm{~m}$, while for $\theta$ $=60$ is $2.4 \mathrm{~m}$. Or consider the dual case, where the $\mathrm{H}$ is actually $1.88 \mathrm{~m}$, then the calculated range for $\theta=50$ is $4.85 \mathrm{~m}$ and for $\theta=60$ is $4.36 \mathrm{~m}$. This highlights the dependences of the track height on tracker angle.

To put this into context, the goal of a tracker operation is to reduce the track split. The average blade height says something about $\beta$, the conning angle of the rotor system, but it's the individual blade track deviation which is of interest.

For example, the actual blade track heights for the blades are: $1.8833327,1.8772759,1.8802974$, and 1.8814026 , then the track split is $6.06 \mathrm{~mm}$. For this example, because of the unknown angle $\theta$, the range of tack split could between: 5.31 and $8.54 \mathrm{~mm}$, or an error of -12 to $+28 \%$.

\subsection{Using the third pulse for Range}

The EUTD outputs a third pulse, which is the time of the blade trailing edge passing the $\mathrm{d} 2$ diode. If we define this time as $T_{23}$, then given a known blade cord width, the blade radius would be:

$$
\text { radius }=\operatorname{cord} / T_{23} \times \Omega
$$

This gives a calculation of radius that is not dependent on the tracker mount angle, $\theta$. While blades do tapper, this is toward the very end of the blade. For the range was which the tracker see the blade, the chord is relatively constant. At a range of $4.475 \mathrm{~m}$ and less, the cord was $.27 \mathrm{~m}$. From $4.475 \mathrm{~m}$ to $4.8 \mathrm{~m}$, the cord decreased to $.255 \mathrm{~m}$, and from $4.8 \mathrm{~m}$ to the end of the blade, the cord decreases to $19.5 \mathrm{~mm}$.

In general, the main rotor for the Bell 407 rotates at 413 RPM, or an $\Omega$ of 43.2493 , with a blade cord of $0.27 \mathrm{~m}$, an average $T_{23}$ of 0.0013 seconds, gives a blade range of $4.65 \mathrm{~m}$. Using eq 8., one can see that the maintainer mounted the tracker at a $\theta$ of 54 degrees.

At $\theta$ of 50 , for this example, the blade range would be: $4.84 \mathrm{~m}$ with a cord of $0.255 \mathrm{~m}$. Using a configuration cord of $0.27 \mathrm{~m}$, when if fact the cord is 0.255 would give the maximum error, or a track split (in this example) of $5.89 \mathrm{~mm}$ vs $6.06 \mathrm{~mm}$, or an error of $-2.7 \%$. This is significantly less that using only a two pulse system.

\section{Flight Test Data}

Recently, in the evaluation for supplemental type certificate (STC) of a health and usage monitoring system for a Bell 407GX, tracker data was collected for system evaluation. A track acquisition was taken at 100 knots. The blade track is given by blade color in Table 1: Blue, Orange, Red, and Green.

\begin{tabular}{|lll|}
\multicolumn{2}{c}{ Table 1 Blade vs. Track Height and Lead/Lag } \\
BLADE & $\begin{array}{l}\text { TRACK } \\
\text { HEIGHT MM }\end{array}$ & $\begin{array}{l}\text { LEAD/LAG } \\
\text { DEGREES }\end{array}$ \\
\hline BLUE & 2.7555 & 0.0296 \\
\hline ORANGE & -3.3012 & 0.0135 \\
\hline RED & -0.2798 & 0.0214 \\
\hline GREEN & 0.8254 & -0.0645 \\
\hline
\end{tabular}

While the track height for the blades was approximately $1.88 \mathrm{~m}$, the data is displayed by removing the mean track height value, with the remainder in $\mathrm{mm}$. The lead/lag is also measured.

This data is the average tack height and lead/lag over a five second period. While important for maintenance, much insight into the rotor dynamics can be gained by looking at the evolution of the track height and lead/lag over time (Figure 6).

As can be seen in both figure 4 (main rotor RPM) and figure 6 , the dynamics of the rotor system are complex. The 
change in group rotor blade tip height relates to the change in shaft angle and feathering control of the helicopter (Gessow, Myers, 1967).
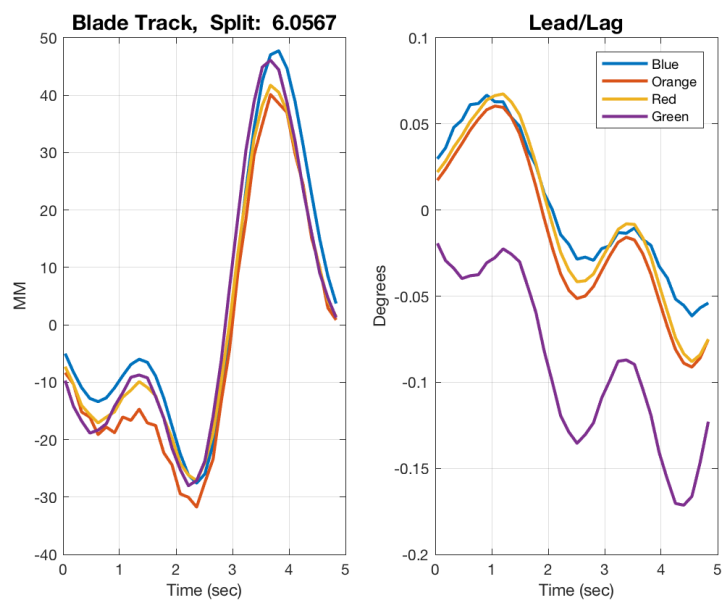

Figure 7 Track Height and Lead/Lag over 5 seconds

This type of information is of great interest to rotor dynamists and may better define control logic for fly by wire aircraft.

\section{Conclusion}

Rotor Track and Balance, as part of the health and usage monitoring system (HUMS), adds considerable value to a customer. In the development of a HUMS for the Bell 407, we found no analysis comparing two pulse v.s three pulse optical tracker. Further, while a number of papers concerning the optimization of track split have been published, there was no description of how track blade height is calculated. As such, this paper was the result of research in the to development of the Track blade height calculation with the EUTD (Enhanced Universal Tracking Device).

Because of how the tracker is mounted on the aircraft, there is always the possibility that the configuration range of the blade needed in track calculation is different from the actual range, resulting in blade height calculation error. This is the case of traditional, two pulse trackers. The use of a third pulse in the EUTD, allows the use of blade chord width to better estimate blade range. This in turn reduces the possible error of the height calculation. For the flight test data, the potential error in the system was reduced for a range of -12 to $+28 \%$ using a two pulse system, to $-2.7 \%$ to 0 error.

\section{REFERENCES}

Aviation Pros. (1999), Helicopter track and balance theory, http://www.aviationpros.com/article/10389059/helicopt er-track-and-balance-theory

Bell Helicopter (2014), Chapter 18, Vibration Analysis, BHT-407-MM-2, page 23.

Gessow, A., \& Myers, G., (1967), Aerodynamics of the Helicopter, Co, New York, Frederic Ungar Publishing, page 138-179.

Hadley, M., (1993) Rotor blade tracking devices, US Patent, $5,249,470$

Veca, A., (1973). Vibration Effects on Helicopter Reliability and Maintainability. U.S. Army Air Mobility Research and Development Laboratory Technical Report 73-11.

\section{BIOGRAPHIES}

Eric Bechhoefer received his B.S. in Biology from the University of Michigan, his M.S. in Operations Research from the Naval Postgraduate School, and a Ph.D. in General Engineering from Kennedy Western University. His is a former Naval Aviator who has worked extensively on condition based maintenance, rotor track and balance, vibration analysis of rotating machinery and fault detection in electronic systems.

Brian Tucker Received his B.S. and M.S. in Mechanical Engineering from the University of Tennessee, Knoxville. During his 21 years at Bell Flight, he has worked in structural test, fatigue analysis, and integrated vehicle health management. 